\title{
Aportes sociológicos en el diálogo interdisciplinario sobre la música
}

[Sociological contributions in the interdisciplinary dialogue on music]

\section{Sonia María Ruiz-Cejudo ${ }^{\mathrm{a}}$}

a Centro de Estudios Sociológicos, Facultad de Ciencias Políticas y Sociales (FCPyS), Universidad Nacional Autónoma de México (UNAM), Circuito Mario de la Cueva s/n, Ciudad Universitaria, C.P. 04510, Coyoacán, Ciudad de México, Distrito Federal, México

Correspondencia:

${ }^{\S}$ Sonia María Ruiz-Cejudo

Circuito Mario de la Cueva s/n, Ciudad Universitaria, C.P. 04510

Centro de Estudios Sociológicos, Facultad de Ciencias Políticas y Sociales (FCPyS),

Universidad Nacional Autónoma de México (UNAM)

Correo-e: smrc308@gmail.com (S.M. Ruiz-Cejudo) 


\title{
Resumen
}

La vida social se construye, entre otros aspectos, por una atmósfera sonora que adquiere forma con base en contextos culturales específicos. Dentro de ella, para muchos grupos humanos la actividad musical ha resultado ser un elemento incluso ritual y que puede llegar a constituir discursivamente sus identidades; para otras, por el contrario, la propia noción de música -y las implicaciones que ésta tiene- para designar muchas de sus prácticas sonoras no existe. ¿Cómo se posiciona la sociología frente a esta dualidad de percepciones sobre "sonido humanamente organizado"? ¿con qué herramientas cuenta un sociólogo para poder indagar y problematizar el fenómeno? El objetivo de este trabajo es presentar elementos que ayuden a responder dichas interrogantes, exponiendo dos posturas epistemológicas propias de la sociología a partir de las que pueden construirse puentes de discusión con la etnomusicología y otras disciplinas, y propiciar diversos acercamientos en torno a la música.

\section{Palabras clave}

sociología; música; estructura social; acción colectiva

\begin{abstract}
Social life is built, among other aspects, by a sound atmosphere that acquires shape based on specific cultural contexts. Within it, for many human groups, musical activity has turned out to be an element that is even ritual and can discursively constitute their identities; for others, on the contrary, the very notion of music -and its implications- to designate many of its sound practices, does not exist. How is sociology positioned in front of this duality of perceptions about "humanly organized sound"? What tools does a sociologist have to be able to investigate and problematize this phenomenon? The aim of this work is to present elements that help answer these questions, exposing two epistemological positions of sociology from which can be built bridges of discussion with ethnomusicology and other disciplines, as well as promoting various approaches around music.
\end{abstract}

\section{Key words}

sociology; music; social structure; collective action 


\section{Introducción}

Los seres humanos nos encontramos de manera permanente relacionados con el sonido, pues en conjunto y también a partir de él conocemos, pensamos, nos relacionamos, expresamos, sentimos e imaginamos. En este sentido, juega un papel significativo en nuestra aprehensión y construcción simbólica del mundo, por lo que se vuelve un imperativo que las ciencias sociales aborden sus manifestaciones.

De manera sucinta, frente al sonido humanamente organizado ${ }^{l}$ se despliega una dualidad de percepciones, pues mientras muchos concebimos diversas manifestaciones como musicales, ya sea cantar, asistir a un concierto, tocar un instrumento, tararear de paseo por la calle o escuchar canciones con un reproductor digital, para ciertos grupos sociales -aún en Occidente- la noción propia de música para designar algunas de sus prácticas sonoras, no existe: los "juegos de garganta" así como otras prácticas en las comunidades inuit; el silbo gomero, situado en La Gomera - perteneciente a las islas Canarias- es un lenguaje y no una música, al igual que en otras regiones del globo donde "se traducen" lenguas a silbidos como México o Papúa Nueva Guinea. La recitación del Corán no puede nombrarse como canto, aunque incluso requiere de una enseñanza particular de vocalización, pues resultaría profano dado su contexto; por su parte, la lengua alemana no permite la acepción en plural para el término musik, de tal modo que con él sería imposible hablar de "las músicas del mundo".

Sin embargo, todas estas manifestaciones mantienen una característica en común, que permite distinguirlas y hacerlas asibles para el ojo sociológico: son prácticas situadas en tiempo y espacio, es decir, que están localizadas en contextos particulares que las dotan de sentido. Además, todas ellas se derivan y refieren a otras acciones, significados, relaciones sociales, interacciones, identidades y valoraciones, también referentes a una cultura. El objetivo de este trabajo es problematizar, tanto para científicos sociales como para interesados en el tema, cómo analiza la sociología estos elementos para entender y explicar la experiencia musical como constructo social.

Así, para sintetizar las potencialidades teóricas y las herramientas propias de la tradición sociológica en su construcción de la música como objeto de estudio presento dos

\footnotetext{
${ }^{1}$ Concepto elaborado por el etnomusicólogo John Blacking, citado por Pelinski (2000)
} 
posturas epistemológicas que se han desarrollado en ella y que son distinguibles en la pregunta ¿la estructura social hace a los individuos o los individuos construyen la sociedad?. Ésta se ha desarrollado como un amplio debate en nuestra disciplina en relación con cómo podemos entender la realidad social, por lo que con ambos enfoques busco realizar una sistematización de los elementos que permean las diversas corrientes de pensamiento que, hasta la actualidad, se han ocupado de estudiar el carácter social de la música.

Por un lado, está el enfoque que pondera el estudio del carácter estructural de los fenómenos sociales. En estos términos, el análisis está centrado en los rasgos institucionalizados, dando énfasis a cómo las condiciones históricas permean, e incluso pueden dominar e inhabilitar al sujeto; de este modo, lo social se plantea como algo externo y coercitivo. En términos generales, las interrogantes que responde esta corriente se refieren principalmente a localizar y medir regularidades, así como a explicar causalidades de hechos sociales.

$\mathrm{Y}$ en segundo lugar, está el que reivindica el papel del individuo como agente constructor de lo social. En este caso el análisis se dirige al sentido de la acción; el sujeto no se ve totalmente constreñido por la estructura pues se reconoce su capacidad reflexiva frente al mundo, con base en la que también puede modificar su conducta. Así, lo social es resultado del obrar humano intencionado y al estudiarlo se busca comprender significados.

A partir de esta distinción expongo de manera general las propuestas de dos autores que problematizan sociológicamente a la música para evidenciar con mayor precisión cómo se desarrollan ambas formas de pensamiento. Si bien, esta dualidad no se presenta siempre de manera tajante y las características discutidas pueden llegar a convivir en el análisis social, sistematizar aquí ejemplos tan contrastantes permite visibilizar la multiplicidad de elementos a partir de los que podemos enfocar un fenómeno y poner en diálogo con otros enfoques y disciplinas para generar nuevas preguntas de investigación.

\section{Teoría Crítica de la música}

Para abordar el énfasis en la estructura social como condicionamiento y elemento fundamental del análisis, considero pertinente mencionar el trabajo de Theodor W. Adorno, 
sociólogo alemán, además de filósofo y músico, que presenció el contexto de las industrias culturales nacientes. Vivió el exilio consecuencia de la Segunda Guerra Mundial y es uno de los principales exponentes de la Teoría Crítica, misma que hace un rescate del marxismo y origina un encuentro multidisciplinario entre sociología, psicoanálisis, filosofía y economía.

En dicha construcción multidisciplinaria la escuela de Frankfurt realiza un análisis de la sociedad moderna como un engranaje entre la organización económica capitalista y el creciente desarrollo tecnológico, produciendo diversas formas de dominación que, desplegadas para crear una falsa conciencia, se basan en una cultura de masas que estandariza las formas comunicativas -incluyendo a la música, según plantea Adorno- y crean necesidades falsas. En este contexto, reina la racionalidad instrumental y hay un poderoso auge de la individualización; el progreso científico y técnico se convierten en una herramienta de dominación que lleva a la máxima contradicción de la sociedad industrial: la irracionalidad de vivir en una aparente satisfacción, producto del creciente dominio del hombre y la naturaleza.

Para Adorno, de manera general, la música como "obra auténtica" tiene características muy específicas: es comunicativa además de expresiva, y se crea de forma autónoma bajo un ideal -con anclaje en el romanticismo-donde el compositor está emancipado de tal modo que su obra aspira a un valor filosófico y que rebasa la mera dimensión comercial, además de mantener una estructura compleja, desafiante e incluso "poco comprensible" a la recepción de un público común. Como consecuencia de estos elementos, su autonomía la conduce al aislamiento y favorece que su aceptación ocurra en las minorías que mantienen cierto refinamiento del gusto musical:

El arte serio se ha negado a aquellos para quienes la miseria y la opresión de la existencia convierten la seriedad en burla y se sienten contentos cuando pueden emplear el tiempo durante el que no están atados a la cadena en dejarse llevar. El arte ligero ha acompañado como una sombra al arte autónomo. Es la mala conciencia social del arte serio. Lo que éste tuvo que perder de verdad en razón de sus premisas sociales confiere a aquél una apariencia de legitimidad. (Horkheimer y Adorno, 1998:180) 
De aquí que la masificación de lo que él llama "música ligera" exprese la irracionalidad propia de la sociedad industrial, al ser recibida como un producto comercial más, en donde cualquier dejo de reflexión y crítica están eclipsados. Así, la producción en serie asigna a la música la única función de mercancía para el entretenimiento y propicia la pérdida de su carácter emancipador.

En consecuencia, la música - como otras expresiones del arte- también se ve supeditada a los efectos del proceso que genera "una amplia estandarización del gusto y de la capacidad de recepción" (Adorno y Eisler, 1976:13-14) de manera que la subordinación del creador a los estándares de comercialización ocasiona la pérdida de su autonomía y la de su obra como algo auténtico, convirtiéndola en una producción calculada como mercancía, pero también en una herramienta de dominación que, mediante el contenido repetitivo, predecible y “comprensible”, perpetúa la satisfacción de un público acrítico y disperso.

Con estos planteamientos es posible reconocer que la estructura de la sociedad tecnológica reprime las libertades individuales mediante una enajenación creciente, de modo que cualquier potencial "revolucionario" presente en el arte autónomo está negado para aquél que sólo es producto de una industria de masas que perpetúa el consumo forzado. En este sentido, si bien se plantean las necesidades individuales y la razón como elementos implicados en el proceso de desarrollo de la sociedad tecnológica, éstos también se ven minimizados por un régimen económico y social que, mediante diversos aparatos de dominación, mantiene una dinámica de simulación donde "a pesar de la abundancia cuantitativa de las ofertas, en realidad la libertad de elección del consumidor es sólo aparente.” (Adorno y Eisler, 1976:14).

Frente a estos aspectos que problematizan las condiciones económicas, sociales e incluso de corte filosófico que rodean al cómo se crea música y de qué manera circula y es recibida por los públicos, es evidente que el análisis se inclina siempre por señalar aquellos elementos que hacen posible la reproducción musical como marcos dados por la historia. Estos marcos, además de permear valores existentes -lo bello, lo creativo, lo necesario, lo que vale la pena pagar-, perfilan prácticas de escucha y de consumo a partir de las que el sistema 
capitalista incorpora y adapta los componentes problemáticos para mantener su existencia.

\section{La música como acción colectiva}

Por otro lado, y para presentar un enfoque dirigido por la reivindicación de la reflexividad e intencionalidad de los sujetos, quiero mencionar el trabajo que realiza Howard S. Becker, sociólogo estadounidense perteneciente a la Escuela de Chicago y músico de jazz rodeado por un contexto donde se desarrollan ideales de democratización y una corriente de pensamiento encaminada en dirección opuesta al funcionalismo reinante de mediados del siglo XX.

A diferencia de Adorno, que concibe al artista y la obra como aspectos especiales y centrales de la música, Becker apunta que el trabajo artístico implica, como el resto de actividades humanas, redes y formas de cooperación que sustentan la existencia de lo que conocemos como obras de arte. En este sentido, su estudio es independiente de cualquier distinción de reputación, pues en todo proceso de creación artística se presentan ideas -materializadas y apoyadas desde diversos puntos-y formas específicas de su recepción.

En este proceso se juegan tanto saberes individuales como saberes compartidos que se despliegan en una multiplicidad de dimensiones para dar existencia a las obras de arte y, generalmente, los patrones de cooperación en los que estos saberes y actividades se comparten, ocurren de manera rutinaria produciendo lo que Becker denomina mundo de arte (Becker, 2008).

Ahora bien, para profundizar en ello específicamente desde el mundo de la música, utiliza el caso de los músicos de jazz, preguntándose ¿cómo se vuelve posible que personas que ni siquiera se conocen puedan tocar juntas toda una noche en lugares públicos? Para resolver esta incógnita es necesario comprender cómo se articulan esos saberes individuales y compartidos que les permiten a los músicos integrar redes de colaboración, generando posibilidades de improvisar y tomar decisiones sobre el escenario cada noche.

En dicha dinámica, sus conocimientos se hacen latentes por un contexto que les permite compartir destrezas desarrolladas tanto en términos teóricos musicales como para resolver problemas durante su interpretación, que además terminan por cristalizarse en la 
configuración de repertorios individuales y colectivos acordes con las experiencias que cada uno adquiere en su trayectoria musical. En el desarrollo de esas experiencias, los músicos de jazz aprenden y construyen sobre el escenario una diversidad de tácticas de negociación para poder tocar en conjunto de manera exitosa piezas que conocen y pueden ir dominando, pero además para explorar ampliamente sus posibilidades o aprender temas nuevos:

Los lugares en que trabajan los músicos tienen sus respectivas exigencias con características propias, requisitos sobre lo que se puede tocar, lo que se debe tocar y lo que no. Cuando la situación los enfrenta a esas características de la demanda, los músicos aprenden canciones que de otro modo quizá no habrían aprendido y aprenden a tocarlas en formas que de otro modo quizá no habrían tocado. (Faulkner y Becker, 2011:61)

Con base en este contexto, para los músicos es posible ir generando rutinas y patrones de cooperación para la interpretación porque, a pesar de presentarse en escenarios con demandas musicales versátiles, esa misma diversidad de experiencias y aprendizajes también los lleva a descubrir y desarrollar sus habilidades y conocimientos para aprender a dominar y sentirse cómodos tocando diversos estilos.

Sin embargo, no hay que olvidar que para que estas actividades tengan lugar es necesaria la acción de otros individuos, previamente y durante el proceso: la fabricación de instrumentos musicales, la limpieza y adecuación del escenario, la transcripción y distribución de textos musicales para que los temas puedan circular y ser aprendidos, las grabaciones y transmisiones para escuchar por primera vez una pieza desconocida, entre otras, son sólo algunas de las condicionantes para perpetuar el mundo de la música en las que, ni el intérprete ni el creador realizan directamente tarea alguna.

Más que un interés por estilos o por valores estéticos, Becker dirige su análisis en torno a la organización social que subyace al trabajo artístico, donde además del creador se ven involucrados los intérpretes y sus maestros, oyentes que asisten al concierto y que son consumidores de discos y otros productos, dueños de establecimientos que albergan los conciertos, intendentes y ayudantes para preparar el escenario, promotores de bailes y bailarines asistentes, ingenieros de audio, constructores de instrumentos, productores 
artísticos, entre otros, que hacen posible su aparición de maneras muy específicas (Figura 1). Así, desde este enfoque se evidencia que de las acciones concretas y los sentidos impresos por los actores en el curso de las relaciones cotidianas que establecen -incluso de aquellos que están ocultos a simple vista- depende la existencia de la obra artística.

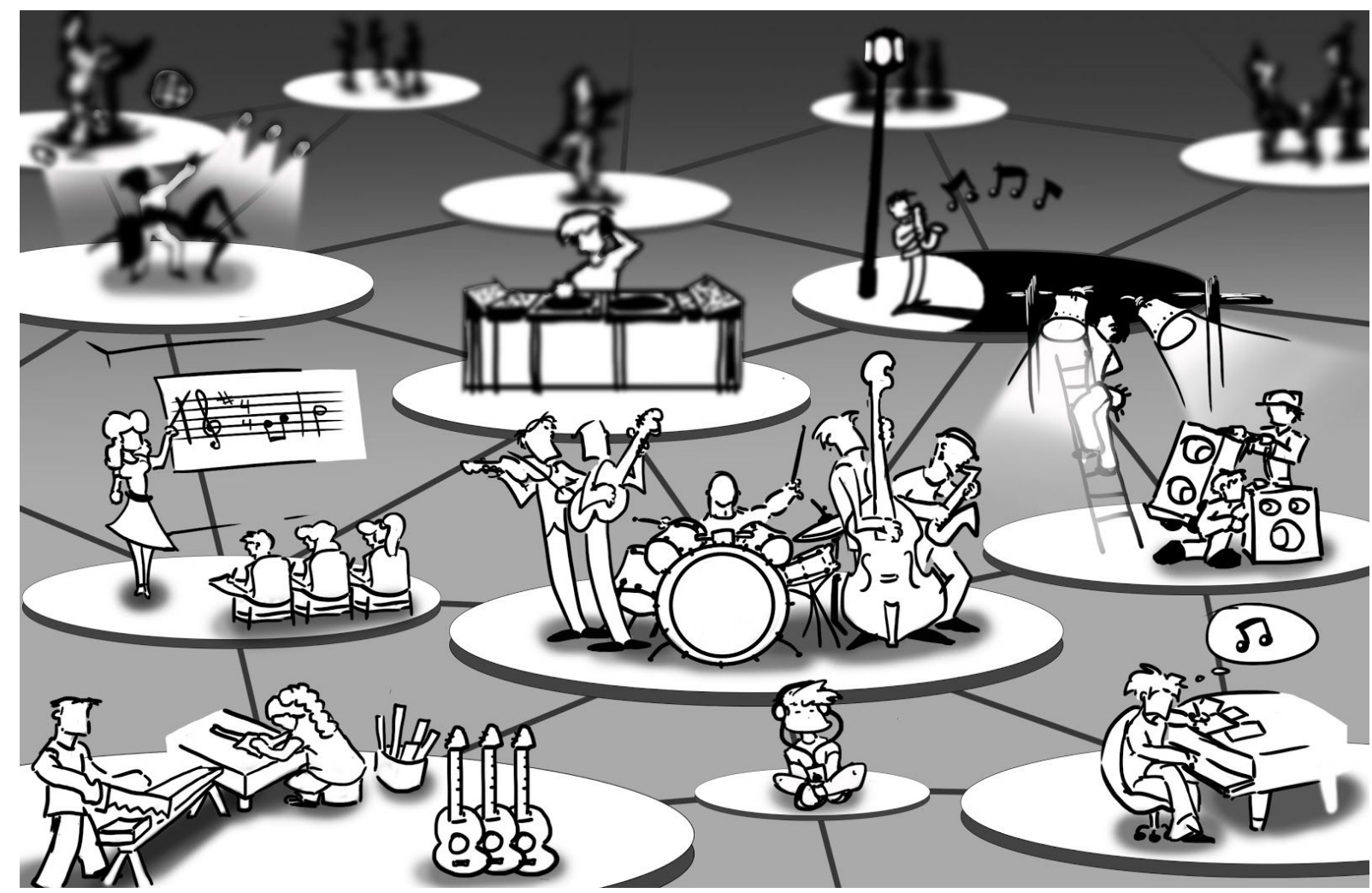

Figura 1. Estructura social conformada por redes de cooperación y prácticas que sustentan el desarrollo del fenómeno musical. En la imagen podemos apreciar roles sociales diversos involucrados en la reproducción del mundo de la música: los intérpretes, el compositor, el ingeniero de audio, el staff, los lauderos, los maestros, el oyente, los bailarines, entre otros que pueden parecer lejanos a simple vista, como productores o representantes.

Además de éstas, sociológicamente se han desarrollado muchas otras interrogantes en la tarea de conceptualizar la música y comprender los factores que condiciona y por los que se configura. Con base en los dos ejemplos discutidos en este trabajo es posible localizar las potencialidades que el enfoque sociológico ofrece para el estudio de los fenómenos sociales, y en particular, del musical. Los conceptos y aspectos generales de esta disertación fueron extraídos y sintetizados mediante minería de texto, de manera que el lector tenga un esquema general visual del abordaje aquí planteado (Figura 2). En ella es posible observar la presencia de elementos micro y macrosociológicos tales como acción, saberes, cultura y sociedad, entre 
otros. En la imagen podemos apreciar como conceptos centrales del texto a la música y lo social, donde presentando los trabajos de Adorno y Becker podemos abordar la acción y el sentido, así como la estructura y el contexto, rodeados por aspectos tales como valoraciones, experiencias, entre otros.

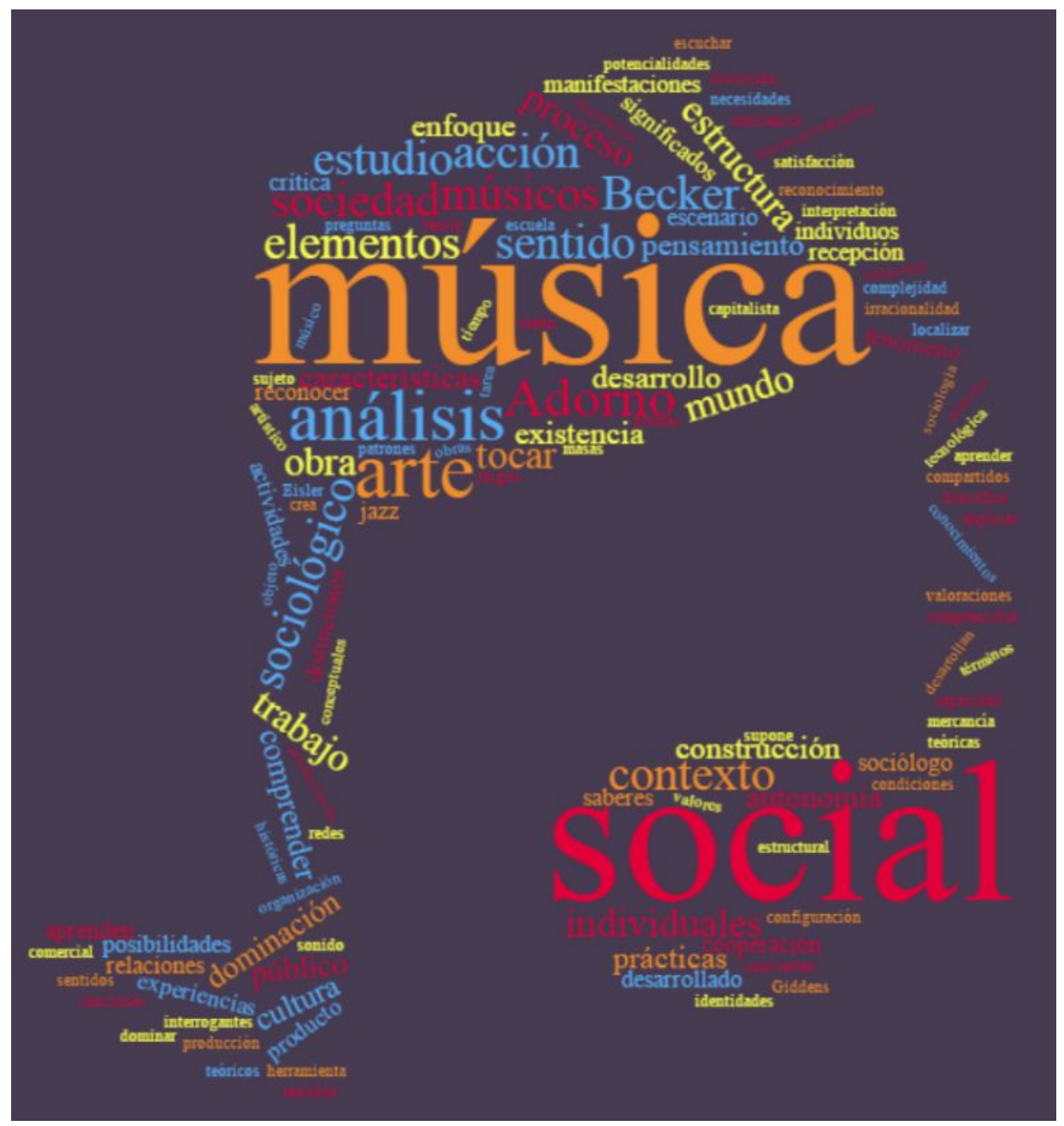

Figura 2. Aspectos sociológicos en el diálogo interdisciplinario sobre la música. Los términos contenidos dentro de dos corcheas sintetizan este trabajo mediante minería de texto. El tamaño de las palabras es proporcional a su frecuencia, abordando la acción y el sentido, la estructura y el contexto referentes a la música y lo social.

De manera sucinta, lo que podemos reconocer en sociología para abordar éste y otros fenómenos es: la capacidad de construir desde distintos niveles de discusión al objeto de estudio; la construcción de distinciones conceptuales; un análisis crítico; argumentación históricamente fundada; así como construcciones teóricas e investigación empírica. Partiendo de este reconocimiento y de la complejidad que supone el espectro social, quiero enfatizar la relevancia de ambos enfoques expuestos recuperando la propuesta del sociólogo británico 
Anthony Giddens (Giddens, 2003) en que plantea la necesidad de reconocer ambas dimensiones para lograr una comprensión profunda de lo social mediante la síntesis entre rasgos sociales institucionalizados y sentidos articulados en copresencia por los actores.

La etnomusicología, al estar permeada por elementos teóricos y herramientas de investigación de las ciencias sociales, puede encontrar múltiples puentes de discusión con la sociología. A partir de ellos será posible generar nuevas preguntas alrededor del fenómeno musical que integren un reconocimiento más amplio de la complejidad que supone el entorno social para su desarrollo, así como el papel de la música en la configuración de sentidos, valoraciones, identidades, significados y relaciones culturales.

\section{Conclusión}

Ya sea ponderando el análisis sobre el carácter estructural de las prácticas sociales o reconociendo la importancia que tienen las narrativas biográficas para explicar el mundo, la sociología ha desarrollado enfoques diversos para comprender la música como un fenómeno multifactorial y que es permeado por la cultura. De estas aportaciones teóricas en las que la música puede entenderse como una estructura o un hacer individual, se desprende la posibilidad de establecer discusiones interdisciplinarias fructíferas en torno a su complejidad.

\section{Agradecimientos}

SMRC agradece a Carlos Saldaña Cejudo (carlos_cscx@hotmail.com) por su ayuda en el diseño de la figura 1, y a José Darío Martínez Ezquerro por su ayuda con la minería de texto de la figura 2. 


\section{Bibliografía}

Adorno, Theodor W. y Eisler, Hanns (1976). El cine y la música. Madrid: Editorial Fundamentos

Becker, Howard (2008). Los mundos del arte. Argentina: Universidad Nacional de Quilmes

Faulkner, Robert y Becker, Howard (2011). El jazz en acción. La dinámica de los músicos sobre el escenario. Buenos Aires: Siglo XXI Editores

Giddens, Anthony (2003). La constitución de la sociedad. Bases para la teoría de la estructuración. Buenos Aires: Amorrortu

Horkheimer, Max y Adorno, Theodor W. (1998). Dialéctica de la Ilustración. Madrid: Editorial Trotta

Pelinski, Ramón (2000). Invitación a la etnomusicología. Quince fragmentos y un tango. Madrid: Akal 